\title{
Reducing Radiation Dose in Adult Head CT using Iterative Reconstruction - A Clinical Study in 177 Patients
}

\section{Dosisreduktion bei der cranialen CT mit Hilfe iterativer Rekonstruktion - eine klinische Studie an 177 Patienten}

Authors

Affiliations

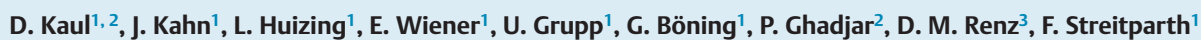

Department of Radiology, Charité School of Medicine and University Hospital, Berlin, Germany

Department of Radiation Oncology, Charité School of Medicine and University Hospital, Berlin, Germany

3 Department of Radiology, Jena University Hospital, Jena, Germany
Keywords

cranial computed

tomography

cCT

- iterative reconstruction

- ASIR

- dose reduction

- filtered back projection received 25.5.2015

accepted 1.9.2015

\section{Bibliography}

Dol http://dx.doi.org/

10.1055/s-0041-107200

Published online: 3.11.2015

Fortschr Röntgenstr 2016; 188:

155-162 (c) Georg Thieme

Verlag KG Stuttgart · New York ·

ISSN 1438-9029

\section{Correspondence \\ Dr. David Kaul}

Department of Radiation Oncology, Charité School of

Medicine and University

Hospital

Charitéplatz 1

10117 Berlin

Germany

Tel.: ++49/30/450527152

Fax: ++49/30/4507527152

david.kaul@charite.de

\section{Zusammenfassung \\ $\nabla$}

Ziel: Es war das Ziel dieser Studie zu untersuchen, inwiefern die adaptive statistische iterative Rekonstruktion (ASIR) zur Dosisreduktion bei der nativen, craniellen Computertomografie (cCT) beitragen kann und welchen Einfluss sie auf die Bildqualität hat.

Material und Methoden: Es wurden 177 native cCT unter Notfallbedingungen durchgeführt. Insgesamt wurden 4 verschiedene Protokolle genutzt: Gruppe A (Kontrollgruppe): $120 \mathrm{kV}$, FBP (filtered back projection) $\mathrm{n}=71$; Gruppe B1: $120 \mathrm{kV}$, Scan und Rekonstruktion durchgeführt mit $20 \%$ ASIR (Überblendung von $20 \%$ ASIR und $80 \%$ FBP), $n=86$; Gruppe B2: Rohdaten aus Gruppe B1 wurden mit einer Überblendung von $40 \%$ ASIR und $60 \%$ FBP rekonstruiert, $n=74$; Gruppe C1: $120 \mathrm{kV}$, Scan und Rekonstruktion durchgeführt mit $30 \%$ ASIR, $n=20$; Gruppe C2: Rohdaten aus Gruppe C1 wurden mit einer Überblendung von of $50 \%$ ASIR and $50 \%$ FBP rekonstruiert, $\mathrm{n}=20$. Die Effektivdosis aller CTs wurde berechnet; die Bildqualität wurde sowohl quantitativ als auch qualitativ evaluiert.

Ergebnisse: Im Vergleich zur Gruppe A zeigten die Gruppen $B 1 / 2$ und $C 1 / 2$ eine signifikant reduzierte Effektivdosis von $40,4 \%$ und $73,3 \%$ ( $p<0,0001$ ). Die Gruppen B1 und die Gruppen C1/2 zeigten dabei eine signifikant verminderte quantitative und qualitative Bildqualität. Die Gruppe B2 zeigte mit der Kontrollgruppe A vergleichbare Werte in der quantitativen Analyse, die qualitative Analyse zeigte schlechtere Werte als die Kontrollgruppe aber bessere Werte als Gruppe B1. Die diagnostische Konfidenz der Gruppen B1/2 war hoch genug für den routinemäßigen Gebrauch in der Klinik. Die diagnostische Konfidenz der Gruppe C2 war hoch genug für Folgebildgebungen bei vordiagnostizierten intracraniellen Blutungen und subakutem Schlaganfall.

\section{Abstract \\ $\nabla$}

Purpose: To assess how ASIR (adaptive statistical iterative reconstruction) contributes to dose reduction and affects image quality of non-contrast cranial computed tomography (cCT).

Materials and Methods: Non-contrast emergency CT scans of the head acquired in 177 patients were evaluated. The scans were acquired and processed using four different protocols: Group A (control): $120 \mathrm{kV}$, FBP (filtered back projection) $\mathrm{n}=71$; group B1: $120 \mathrm{kV}$, scan and reconstruction performed with $20 \%$ ASIR (blending of $20 \%$ ASIR and $80 \% \mathrm{FBP}$ ), $\mathrm{n}=86$; group $\mathrm{B} 2$ : raw data from group B1 reconstructed using a blending of $40 \%$ ASIR and $60 \% \mathrm{FBP}, \mathrm{n}=74$; group C1: $120 \mathrm{kV}$, scan and reconstruction performed with $30 \%$ ASIR, $\mathrm{n}=20$; group C2: raw data from group C1 reconstructed using a blending of $50 \%$ ASIR and $50 \%$ $\mathrm{FBP}, \mathrm{n}=20$. The effective dose was calculated. Image quality was assessed quantitatively and qualitatively.

Results: Compared to group A, groups B1/2 and C1/2 showed a significantly reduced effective dose of $40.4 \%$ and $73.3 \%(\mathrm{p}<0.0001)$, respectively. Group B1 and group C1/2 also showed significantly reduced quantitative and qualitative image quality parameters. In group B2, quantitative measures were comparable to group $\mathrm{A}$, and qualitative scores were lower compared to group A but higher compared to group B1. Diagnostic confidence grading showed groups B1/2 to be adequate for everyday clinical practice. Group C2 was considered acceptable for follow-up imaging of severe acute events such as bleeding or subacute stroke.

Conclusion: Use of ASIR makes it possible to reduce radiation significantly while maintaining adequate image quality in non-contrast head CT, which may be particularly useful for younger patients in an emergency setting and in follow-up. 
Schlussfolgerung: ASIR bietet die Möglichkeit zur signifikanten Reduktion der Effektivdosis von craniellen CT bei ausreichend hoher Bildqualität für den täglichen klinischen Gebrauch. Dies ist insbesondere bei jungen Patienten und bei mehrfachen Folgeuntersuchungen von Vorteil.

Kernaussagen:

- Durch ASIR kann die Dosisexposition bei klinisch adäquater Bildqualität signifikant vermindert werden

- $20 \%$ ASIR cCT mit 40\%ASIR/60\%FBP Überblendung sind ausreichend für täglichen klinischen Gebrauch

- $30 \%$ ASIR cCTs mit 50\%ASIR/50\%FBP Überblendung sind ausreichend für Folgebildgebungen
Key Points:

- ASIR may reduce radiation significantly while maintaining adequate image quality

- cCT protocol with $20 \%$ ASIR and $40 \%$ ASIR/60\%FBP blending is adequate for everyday clinical use

- cCT protocol with 30\% ASIR and 50\%ASIR/50\%FBP blending is adequate for follow-up imaging

Citation Format:

- Kaul D, Kahn J, Huizing L etal. Reducing Radiation Dose in Adult Head CT using Iterative Reconstruction - A Clinical Study in 177 Patients. Fortschr Röntgenstr 2016; 188: 155-162

\section{Introduction}

$\nabla$

The use of computed tomography (CT) has been constantly increasing over the last decades and leads to higher cumulative doses of ionizing radiation in the population [1]. According to a recent survey conducted by the German Agency for Radiation Protection, CT examinations constitute $8 \%$ of all radiological examinations and account for $63 \%$ of the total population dose due to radiological examinations [2].

With the widespread availability of CT scanners, emergency departments have seen a remarkable increase in the use of cranial CT (cCT). In an emergency setting, non-contrast cCT is usually performed to rapidly rule out intracranial pathology. However, many patients who undergo emergency cCT have no intracranial pathology at all. Furthermore, there is growing evidence that the increasing use of $\mathrm{CCT}$ in younger patients will lead to a higher rate of brain cancer in the future [3].

One of the principles of modern radiology is to apply the lowest possible amount of ionizing radiation while maintaining diagnostic image quality. Efforts made to reduce overall radiation exposure have led to new technologies, such as automated tube current modulation and noise reduction filters $[4,5]$.

Unfortunately, dose reduction with both techniques is limited when a head with a thick skull bone is examined [6]. Lowering the tube potential in the acquisition of $\mathrm{CCT}$ scans reduces radiation effectively but comes at the cost of increased image noise [7]. With the recent developments in computing power, iterative reconstruction (IR) algorithms, which were first introduced for single-photon emission computed tomography (SPECT) and positron emission tomography (PET), can now also be applied to CT $[8,9]$. IR algorithms eliminate some of the increased image noise resulting from the use of a lower tube current for the acquisition of CT scans.

Pilot studies have shown that IR algorithms have the potential to reduce the radiation dose of cranial CT scans by $20-45 \%$ [10-13].

This clinical study analyzes the effect of IR on effective radiation doses, image quality and interpretability in comparison with routine CT scans of the head based on filtered back projection (FBP) in a large patient population examined in an emergency setting.

\section{Materials and Methods \\ $\nabla$}

\section{Study Design}

The institutional ethics board approved this study. Since patients were not exposed to additional radiation and their data were stored anonymously, the informed consent requirement was waived. Five protocols - A, B1, B2, C1 and C2 - with increasing dose reduction potential were used.

Patients in group A and group B1/B2 were referred from the first-aid department. Patients in group $\mathrm{C} 1 / 2$ had undergone head CT before and were referred by the neurosurgical intensive care unit (ICU) for follow-up CT. We did not use protocol C1/2 (protocol with highest dose reduction potential) on first-aid patients to avoid the risk of having to repeat the CT examination due to insufficient image quality.

Most patients underwent cCT for one of the following acute events: trauma and/or amnesia, skull fracture, loss of consciousness, seizure, headache, vomiting, focal neurological deficit, coagulopathy, treatment with anticoagulants, increasing frequency of unexplained headaches or new onset of severe or persistent headache.

Intracranial foreign material was considered an exclusion criterion in groups A, B1 and B2 but not in group C1/2, since virtually all neurosurgical ICU patients carry intracranial foreign material.

\section{CT Protocol}

Protocols are summarized in $\bullet$ Table 1. All patients were examined on a 64-slice multi-detector CT scanner (Lightspeed VCT, GE Healthcare, USA). Patients were scanned at $120 \mathrm{kV}$ and a tube current range of 100-300 mA. Tube current modulation was used. In all cases, images were acquired in a craniocaudal direction.

A control group of 71 patients was scanned using CT protocol A ( $120 \mathrm{kV}$, filtered back projection (FBP), NI: $2.8=$ reference NI). 86 patients were scanned using CT protocol B1 $(120 \mathrm{kV}, 20 \%$ ASIR, NI: 4). By default, the use of $20 \%$ ASIR results in a tube current reduction of approximately $20 \%$. The raw data are analyzed using the FBP and the ASIR algorithms, resulting in blended images of $20 \%$ ASIR and $80 \%$ FBP. In group B2, raw data from group B1 were blended using $40 \%$ ASIR and 60\% FBP. Due to technical reasons, only 74 of 86 patients could be re-blended for group B2. In group C1, 30\% ASIR was used on 20 patients $(120 \mathrm{kV}, 30 \%$ ASIR,

Table 1 CT protocol characteristics.

Tab. 1 Charakteristika der verwendeten Protokolle.

\begin{tabular}{|lcllll|} 
& group A & group B1 & group B2 & group C1 & group C2 \\
\hline $\begin{array}{l}\text { tube } \\
\text { potential }\end{array}$ & $120 \mathrm{kV}$ & $120 \mathrm{kV}$ & $120 \mathrm{kV}$ & $120 \mathrm{kV}$ & $120 \mathrm{kV}$ \\
\hline noise index & 2.8 & 4 & 4 & 6 & 6 \\
\hline ASIR & $0 \%$ & $20 \%$ & $20 \%$ & $30 \%$ & $30 \%$ \\
\hline $\begin{array}{l}\text { blending } \\
\text { ratio }\end{array}$ & $100 \% \mathrm{FBP}$ & $80 \% \mathrm{FBP}$ & $60 \% \mathrm{FBP}$ & $70 \% \mathrm{FBP}$ & $50 \% \mathrm{FBP}$ \\
\hline
\end{tabular}


NI: 6). In group C2, raw data from group C1 were blended using $50 \%$ ASIR and $50 \%$ FBP.

\section{Data Reconstruction}

ASIR is an algorithm-based protocol for reconstructing CT images with a focus on noise reduction. It uses the information obtained from the FBP algorithm as a basis for further transformation. The values of each pixel $(y)$ are transformed using matrix algebra to obtain a new estimate of the pixel value ( $\left.y^{\prime}\right)$, which is then compared with the ideal value predicted by the noise model. Iterative steps are performed until the final estimated and the ideal pixel values ultimately converge [8]. This method allows for selective subtraction of noise from a CT image.

The tool traditionally used to define desired image quality in the user interface in GE scanners is called the noise index (NI). The NI is referenced to the HU standard deviation in a specific size water phantom, which is compared to the attenuation measured in the CT scout. Lowering the noise index leads to lower noise but requires a higher tube current.

When using ASIR, however, a second option to modify tube current is introduced. In a first step the operator choses the level of ASIR in $10 \%$ increments from $0 \%$ to $50 \%$.

By default, the use of $\mathrm{X} \%$ ASIR results in a tube current reduction of approximately $\mathrm{X} \%$ during the scan. Obviously it is possible to choose values for NI and ASIR which mutually exclude each other: e. g. a very low NI and a high level of ASIR or vice versa. In such cases of conflicting NI and ASIR values, the system prioritizes the NI over ASIR. This means that ASIR cannot modify tube current when an insufficient NI is chosen. When the noise index is increased, tube reduction may be higher than expected based on the level of ASIR chosen.

After the scan, raw data are reconstructed alternately using ASIR and FBP. ASIR- and FBP-reconstructed images are then combined in a ratio of X\% ASIR and 100-X\% FBP - e.g. when using $20 \%$ ASIR, tube current is reduced approximately by $20 \%$, raw data are reconstructed using ASIR and FBP and finally images are blended using 20\% ASIR and 80\% FBP. However, after image acquisition different blending ratios can be used (as we have done in groups B2 and $\mathrm{C} 2$ ).

\section{Image Quality}

Image quality was assessed quantitatively and qualitatively. Quantitative image quality was evaluated as signal attenuation (SI) measured in Hounsfield units (HU) and noise (i. e., standard deviation (SD) of attenuation). We chose regions of interest (ROIs) in the lentiform nucleus (ROI1), frontal white matter (WM) (ROI2), frontal cortical layer (ROI3), ventricle (ROI4), internal capsule (ROI5), cortical layer of cerebellum (ROI6), WM of middle cerebellar peduncle (ROI7) and vermis (ROI8) for analysis (॰ Fig. 1).

The signal-to-noise ratio (SNR) was calculated according to the following equation:

$\mathrm{SNR}=\frac{\mathrm{SI}_{\text {ROIa }}}{\left(\mathrm{SD}_{\text {ROIa }}\right)}$

The contrast-to-noise ratio (CNR) was calculated according to the following equation:

$\mathrm{CNR}=\frac{\Delta\left(\mathrm{SI}_{\mathrm{ROIa}}, \mathrm{SI}_{\mathrm{ROIb}}\right)}{\sqrt{\left(\mathrm{SD}_{\mathrm{ROIa}}\right)^{2}+\left(\mathrm{SD}_{\mathrm{ROIb}}\right)^{2}}}$

CNRs were calculated in the supratentorial (ST) region between ROI3/ROI2 (ST-CNR C/WM) and between ROI1/ROI2 (ST-

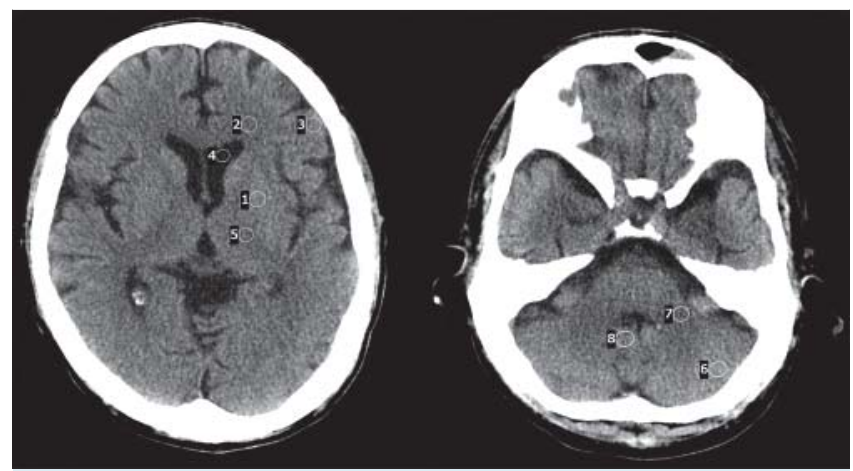

Fig. 1 Sites of ROIs for quantitative image analysis. Supratentorial ROIs included the lentiform nucleus (ROI1), frontal white matter (ROI2), temporal cortical layer (ROI3), ventricle (ROI4) and internal capsule (ROI5). Infratentorial ROls included the cortical layer of the cerebellum (ROI6), WM of the middle cerebellar peduncle (ROI7) and the vermis (ROI8).

Abb. 1 Lage der ROls für die quantitative Bildanalyse. Supratentorille ROIs: Nucleus lentiformis (ROI1), frontale weiße Substanz (ROI2), temporaler Cortex (ROI3), Ventrikel (ROI4) und Capsula interna (ROI5). Infratentorielle ROIs: Cerebellärer Cortex (ROI6), weiße Substanz mittlerer cerebellärer Pedunkel (ROI7) und Vermis (ROI8).

CNR NL/WM). For the infratentorial (IT) CNRS we chose ROI6/ ROI7 (IT-CNR C/WM) and ROi8 / ROI7 (IT-CNR V/WM).

Two experienced radiologists with 5 and 11 years of experience performed qualitative analysis of the acquired images in a blinded fashion after a joint training session. All technical information was removed from the images to reduce expectation bias. Image quality was evaluated in seven categories: noise, supratentorial contrast between cortex and white matter, supratentorial contrast between lentiform nucleus and internal capsule, infratentorial contrast between cortex and white matter, artifacts, overall diagnosability and diagnostic confidence (in patients with diagnosed acute pathology). Each category was evaluated using a five-point Likert scale where the reference was an "ideal exam": 1: non-diagnostic image quality, 2 : uncertainty about the evaluation, 3: restricted assessment, 4: unrestricted diagnostic image evaluation possible, 5: excellent image quality.

\section{Radiation Dose}

Dose-length products (DLPs) and the computed tomography dose index (CTDIvol) were acquired. The effective dose (mSv) was estimated by multiplying the dose-length product by a conversion factor of $0.0021 \mathrm{mSv} \times \mathrm{mGy}^{-1} \times \mathrm{cm}^{-1}[14]$.

\section{Statistical Analysis}

The data were analyzed using GraphPad Prism version $5.0 \mathrm{f}$ for Mac (GraphPad Software, San Diego, California, USA) and IBM SPSS Statistics 19 (New York, USA). Continuous data were analyzed using the Student's t-test, and ordinal data were analyzed using the Mann-Whitney U-test. A p-value of less than 0.05 was considered statistically significant. Interobserver agreement between the two readers was assessed using the Cohen's kappa test. 


\section{Results}

$\nabla$

\section{Patient Characteristics}

Patient characteristics are summarized in $\bullet$ Table 2 . The groups were well balanced in terms of age, male-to-female ratio or cranial diameter.

Of the 157 patients referred for cranial CT scans from the emergency department (groups A and B), $22.3 \%$ showed acute or subacute pathologies, such as acute bleeding or subacute ischemia and
23.6\% showed chronic pathologies (status post-tumor resection, postischemic scarring), and $54.1 \%$ had no pathology ( $\Theta$ Table 2 ). Of the 20 neurosurgical ICU patients referred for follow-up imaging (group C), $75 \%$ showed acute bleeding, $5 \%$ showed subacute ischemia, $5 \%$ had undergone tumor resection, and $15 \%$ were referred due to other pathologies.

\section{Quantitative Analysis of Image Quality}

- Table 3 summarizes the results of quantitative analysis of image quality.

Table 2 Patient characteristics.

Tab. 2 Eigenschaften der Patienten.

\begin{tabular}{|c|c|c|c|c|c|c|}
\hline & overall & group A & group B1 & group B2 & group C1 & group C2 \\
\hline & & $120 \mathrm{kV} / \mathrm{FBP}$ & $120 \mathrm{kV} / A S I R 20$ & $\begin{array}{l}120 \mathrm{kV} \text { |ASIR20 } \\
(40 \% / 60 \%)\end{array}$ & 120 kV|ASIR30 & $\begin{array}{l}120 \mathrm{kV} / \text { ASIR30 } \\
(50 \% / 50 \%)\end{array}$ \\
\hline $\mathrm{n}$ & 177 & 71 & 86 & 74 & 20 & 20 \\
\hline age (y) & $58.3 \pm 19.6$ & $62.1 \pm 18.2$ & $55.2 \pm 21.2$ & $55.9 \pm 20.4$ & $58.4 \pm 15.5$ & $58.4 \pm 15.5$ \\
\hline male to female ratio & $77: 100$ & $32: 39$ & $40: 46$ & $34: 40$ & $5: 15$ & $5: 15$ \\
\hline anteroposterior diameter(cm) & $19.6 \pm 0.93$ & $19.8 \pm 1.0$ & $19.5 \pm 0.82$ & $19.5 \pm 0.82$ & $19.3 \pm 0.87$ & $19.3 \pm 0.87$ \\
\hline transverse diameter $(\mathrm{cm})$ & $15.9 \pm 0.8$ & $16.1 \pm 0.7$ & $15.7 \pm 0.83$ & $15.7 \pm 0.85$ & $15.6 \pm 0.79$ & $15.6 \pm 0.79$ \\
\hline no pathology & $86(48.6 \%)$ & $28(39.4 \%)$ & $57(66.2 \%)$ & $50(67.6 \%)$ & $0(0 \%)$ & $0(0 \%)$ \\
\hline acute bleeding & $35(19.8 \%)$ & $14(19.7 \%)$ & $6(7 \%)$ & $6(8.1 \%)$ & $15(75 \%)$ & $15(75 \%)$ \\
\hline subacute ischemia & $16(9 \%)$ & $9(12.7 \%)$ & $6(7 \%)$ & $5(6.8 \%)$ & $1(5 \%)$ & $1(5 \%)$ \\
\hline post ischemia & $9(5.1 \%)$ & $6(8.5 \%)$ & $3(3.5 \%)$ & $3(4.1 \%)$ & $0(0 \%)$ & $0(0 \%)$ \\
\hline post tumor resection & $16(9 \%)$ & $7(9.9 \%)$ & $8(9.3 \%)$ & $6(8.1 \%)$ & $1(5 \%)$ & $1(5 \%)$ \\
\hline other non-acute pathology & $15(8.5)$ & $7(9.9 \%)$ & $6(7 \%)$ & $4(5.4 \%)$ & $3(15 \%)$ & $3(15 \%)$ \\
\hline
\end{tabular}

The groups were well balanced with respect to age, male/female ratio and cranial diameter. The high number of patients with no intracranial pathology underlines the necessity to keep the level of ionizing radiation as low as reasonably possible.

Die Gruppen waren ausgeglichen in Bezug auf Alter, Geschlechtsverhältnis und Kopfdurchmesser. Die hohe Anzahl an Patienten ohne intracranielle Pathologie unterstreicht die Wichtigkeit die Dosis ionisierender Strahlen so niedrig wie möglich zu halten.

Table 3 Quantitative analysis of image quality.

Tab. 3 Quantitative Analyse der Bildqualität.

\begin{tabular}{|c|c|c|c|c|c|c|c|c|c|}
\hline & group A & group B1 & A vs. B1 & group B2 & A vs. B2 & group C1 & A vs. C1 & group C2 & A vs. $C 2$ \\
\hline & $120 \mathrm{kV} / \mathrm{FBP}$ & 120 kV/ASIR20 & p-value & $\begin{array}{l}120 \mathrm{kV} / \text { ASIR20 } \\
(40 \% / 60 \%)\end{array}$ & p-value & $120 \mathrm{kV} / \mathrm{ASIR} 30$ & $\mathrm{p}$-value & $\begin{array}{l}120 \mathrm{kV} / \text { ASIR30 } \\
(50 \% \mid 50 \%)\end{array}$ & $\mathrm{p}$-value \\
\hline SNR ROI1 & $8.6 \pm 1.4$ & $6.8 \pm 0.97$ & $p<0.0001$ & $7.8 \pm 1.2$ & $p<0.0001$ & $4.4 \pm 0.86$ & $p<0.0001$ & $5.2 \pm 0.87$ & $p<0.0001$ \\
\hline SNR ROI2 & $6.8 \pm 1.3$ & $5.2 \pm 0.73$ & $p<0.0001$ & $5.8 \pm 0.99$ & $p<0.0001$ & $3.8 \pm 0.65$ & $p<0.0001$ & $4.2 \pm 0.84$ & $p<0.0001$ \\
\hline SNR ROI3 & $8.6 \pm 1.4$ & $7.5 \pm 1$ & $p<0.0001$ & $7.7 \pm 1.4$ & $p<0.0001$ & $5.2 \pm 1$ & $p<0.0001$ & $5.9 \pm 1.5$ & $p<0.0001$ \\
\hline SNR ROI4 & $1.2 \pm 0.51$ & $1.0 \pm 0.36$ & $p=0.001$ & $1.1 \pm 0.39$ & $p=0.10$ & $0.57 \pm 0.25$ & $p<0.0001$ & $0.64 \pm 0.34$ & $p<0.0001$ \\
\hline SNR ROI5 & $6.6 \pm 1.2$ & $5.2 \pm 0.9$ & $p<0.0001$ & $5.8 \pm 1.1$ & $p<0.0001$ & $3.3 \pm 0.49$ & $p<0.0001$ & $3.7 \pm 0.44$ & $p<0.0001$ \\
\hline SNR ROI6 & $10.2 \pm 1.6$ & $9.0 \pm 0.14$ & $p<0.0001$ & $10.0 \pm 1.7$ & $p=0.53$ & $7 \pm 1.3$ & $p<0.0001$ & $6.9 \pm 1.3$ & $p<0.0001$ \\
\hline SNR ROI7 & $6.3 \pm 1.1$ & $5.3 \pm 1.0$ & $p<0.0001$ & $5.7 \pm 1.2$ & $p=0.004$ & $4.2 \pm 0.98$ & $p<0.0001$ & $4.4 \pm 8.4$ & $p<0.0001$ \\
\hline SNR ROI8 & $8.5 \pm 1.8$ & $7.4 \pm 1.3$ & $p<0.0001$ & $8.5 \pm 1.3$ & $p=0.91$ & $5.1 \pm 1.1$ & $p<0.0001$ & $5.7 \pm 1.6$ & $p<0.0001$ \\
\hline ST-CNR C/WM & $1.86 \pm 0.5$ & $1.71 \pm 0.4$ & $p=0.05$ & $1.81 \pm 0.42$ & $p=0.5$ & $1.14 \pm 0.47$ & $p<0.0001$ & $1.24 \pm 0.58$ & $p<0.0001$ \\
\hline ST-CNR NL/WM & $1.39 \pm 0.32$ & $1.13 \pm 0.27$ & $p<0.0001$ & $1.47 \pm 0.33$ & $p=0.13$ & $0.66 \pm 0.26$ & $p<0.0001$ & $0.91 \pm 0.23$ & $p<0.0001$ \\
\hline IT-CNR C/WM & $2.69 \pm 0.69$ & $2.19 \pm 0.74$ & $p<0.0001$ & $2.7 \pm 0.66$ & $p=0.94$ & $1.64 \pm 0.52$ & $p<0.0001$ & $1.53 \pm 0.43$ & $p<0.0001$ \\
\hline IT-CNR V/WM & $1.55 \pm 0.56$ & $1.4 \pm 0.55$ & $p=0.08$ & $1.75 \pm 0.5$ & $p=0.026$ & $0.75 \pm 0.34$ & $p<0.0001$ & $0.81 \pm 0.45$ & $p<0.0001$ \\
\hline
\end{tabular}

Compared to group A (control), group B1 showed reduced SNRs and CNRs. Group B2 showed CNRs comparable to group A (except for the infratentorial white matter/vermis CNR). CNR levels were the lowest in group C1. Most SNRs and CNRs increased slightly in group C2 compared to group C1. SNR = signal to noise ratio; CNR = contrast to noise ratio. ST-CNR C/WM = supratentorial CNR (cortex/white matter), ST-CNR NL/WM = supratentorial CNR (lentiform nucleus/white matter), IT-CNR C/WM = infratentorial CNR (cortex/white matter), IT-CNR V/WM = infratentorial CNR (vermis/white matter). Lentiform nucleus (ROI1), frontal white matter (ROI2), frontal cortical layer (ROI3), ventricle (ROI4), internal capsule (ROI5); infratentorial ROIs included the cortical layer of the cerebellum (ROI6), WM of the middle cerebellar peduncle (ROI7) and the vermis (ROI8).

Im Vergleich zur Kontrollgruppe A zeigte Gruppe B1 niedrigere SNRs und CNRs. Gruppe B2 zeigte CNRs, die vergleichbar mit der Kontrollgruppe waren (abgesehen von dem infratentoriellen CNR zwischen weißer Substanz und Vermis). Die CNR-Level waren in der Gruppe C1 am niedrigsten. Die meisten SNRs und CNRs in Gruppe C2 stiegen im Vergleich zur Gruppe C1 leicht an. SNR = Signal/Rausch Verhältnis; CNR = Kontrast/Rausch Verhältnis. ST-CNR C/WM = supratentorielles CNR (Cortex/weiße Substanz), ST-CNR NL/WM = supratentorielles CNR (Nucleus lentiformis/weiße Substanz), IT-CNR C/WM = infratentorielles CNR (Cortex/weiße Substanz), IT-CNR V/WM = infratentorielles CNR (Vermis/weiße Substanz). Supratentorielle ROls: Nucleus lentiformis (ROI1), frontale weiße Substanz (ROI2), frontaler Cortex (ROI3), Ventrikel (ROI4), Capsula interna (ROI5); infratentorielle ROIs: Cerebellärer Cortex (ROI6), weiße Substanz mittlerer cerebellärer Pedunkel (ROI7) und Vermis (ROI8). 
Table 4 Qualitative analysis of image quality and interobserver agreement $\mathrm{k}$.

Tab. 4 Die qualitative Analyse der Bildqualität und Interobserver-Variabilität $\mathrm{k}$.

\begin{tabular}{|c|c|c|c|c|c|c|c|c|c|c|}
\hline & group A & group B1 & A vs. B1 & group B2 & A vs. $B 2$ & group C1 & A vs. C1 & group C2 & A vs. $C 2$ & interobser- \\
\hline & $120 \mathrm{kV} / \mathrm{FBP}$ & $\begin{array}{l}120 \mathrm{kV} / \\
\text { ASIR20 }\end{array}$ & $\mathrm{p}$-value & $\begin{array}{l}120 \mathrm{kV} / \\
\text { ASIR20 } \\
(40 \% \mid 60 \%)\end{array}$ & p-value & $\begin{array}{l}120 \mathrm{kV} / \\
\text { ASIR30 }\end{array}$ & p-value & $\begin{array}{l}120 \mathrm{kV} / \text { ASIR30 } \\
(50 \% / 50 \%)\end{array}$ & p-value & $\begin{array}{l}\text { ver agree- } \\
\text { ment } \mathrm{K}\end{array}$ \\
\hline noise & $3.95 \pm 0.35$ & $3.4 \pm 0.49$ & $p<0.0001$ & $3.74 \pm 0.44$ & $p=0.002$ & $2 \pm 0$ & $p<0.0001$ & $2.3 \pm 0.46$ & $p<0.0001$ & 0.86 \\
\hline $\begin{array}{l}\text { supratentorial } \\
\text { contrast }\end{array}$ & $3.97 \pm 0.33$ & $3.44 \pm 0.48$ & $p<0.0001$ & $3.53 \pm 0.51$ & $\mathrm{p}<0.0001$ & $2 \pm 0$ & $p<0.0001$ & $2.3 \pm 0.46$ & $p<0.0001$ & 0.85 \\
\hline $\begin{array}{l}\text { basal ganglia } \\
\text { contrast }\end{array}$ & $3.87 \pm 0.36$ & $3.47 \pm 0.48$ & $p<0.0001$ & $3.55 \pm 0.47$ & $p<0.0001$ & $2 \pm 0$ & $p<0.0001$ & $2.3 \pm 0.46$ & $p<0.0001$ & 0.86 \\
\hline $\begin{array}{l}\text { infratentorial } \\
\text { contrast }\end{array}$ & $3.82 \pm 0.41$ & $3.43 \pm 0.5$ & $p<0.0001$ & $3.53 \pm 0.5$ & $\mathrm{p}<0.0001$ & $2 \pm 0$ & $p<0.0001$ & $2.3 \pm 0.46$ & $p<0.0001$ & 0.86 \\
\hline artifacts & $5 \pm 0$ & $5 \pm 0$ & $p>0.99$ & $5 \pm 0$ & $p>0.99$ & $5 \pm 0$ & $p>0.99$ & $5 \pm 0$ & $p>0.99$ & $\mathrm{n} / \mathrm{a}$ \\
\hline $\begin{array}{l}\text { diagnosis- } \\
\text { related confi- } \\
\text { dence }\end{array}$ & $5 \pm 0$ & $4.94 \pm 0.17$ & $p=0.11$ & $5 \pm 0$ & $p>0.99$ & $4.55 \pm 0.55$ & $p<0.0001$ & $4.63 \pm 0.54$ & $\mathrm{p}<0.0001$ & 0.72 \\
\hline bleeding & $5 \pm 0$ & $5 \pm 0$ & $p>0.99$ & $5 \pm 0$ & $p>0.99$ & $4.67 \pm 0.48$ & $p<0.0001$ & $4.71 \pm 0.46$ & $p<0.0001$ & 0.81 \\
\hline $\begin{array}{l}\text { subacute } \\
\text { ischemia }\end{array}$ & $5 \pm 0$ & $4.8 \pm 0.42$ & $p=0.067$ & $5 \pm 0$ & $p>0.99$ & $4 \pm 0$ & $p<0.0001$ & $4.5 \pm 0.7$ & $p<0.0001$ & 0.31 \\
\hline $\begin{array}{l}\text { overall diag- } \\
\text { nosability }\end{array}$ & $4.01 \pm 0.26$ & $3.51 \pm 0.47$ & $p<0.0001$ & $3.55 \pm 0.47$ & $p<0.0001$ & $2 \pm 0$ & $p<0.0001$ & $2.3 \pm 0.46$ & $p<0.0001$ & 0.85 \\
\hline
\end{tabular}

Noise, supratentorial and infratentorial contrast were significantly reduced in group B1 and also in group B2, albeit to a lesser extent. Diagnosis-related confidence was not compromised in group B1 or B2. Group C1 showed significantly poorer results in terms of noise levels, contrast, diagnostic confidence and overall diagnosability, compared to groups A and B1/2. Group C2 showed only slightly better results than group C1. The interobserver agreement was excellent (>0.75) for grading image noise, contrast and overall diagnosability and good $(>0.4)$ for overall diagnostic confidence and diagnostic confidence in patients with bleeding. The interobserver agreement in diagnostic confidence in patients with subacute stroke was lower but still acceptable. Interobserver agreement $\mathrm{k}$ cannot be calculated for artifacts because both observers assigned a score of 5 for this parameter to all CTs in all groups.

In Bezug auf Rauschen und supra- bzw. infratentoriellen Kontrast zeigten sich signifikant schlechtere Ergebnisse in Gruppe B1 als auch in Gruppe B2. Das diagnosenspezifische Vertrauen war weder in Gruppe B1 noch in Gruppe B2 eingeschränkt. Gruppe C1 zeigte signifikant niedrigere Werte im Hinblick auf Rauschen, Kontrast, spezifischen diagnostischen Wert und diagnostischen Gesamtwert, verglichen mit den Gruppen A, B1 und B2. Die Gruppe C2 zeigte allenfalls leicht bessere Ergebnisse als die Gruppe C1. Die Interobserver-Variabilität war exzellent (>0.75) in Bezug auf Bildqualität, Rauschen, Kontrast und diagnostischen Gesamtwert, gut (>0.4) in Bezug auf den spezifischen diagnostischen Gesamtwert sowie spezifischen diagnostischen Wert bei intracraniellen Blutungen. Die Interobserver-Variabilität in Bezug auf den spezifischen diagnostischen Wert bei subakutem Schlaganfall war niedriger aber noch akzeptabel. Die Interobserver-Variabilität $\mathrm{K}$ in Bezug auf Artefakte kann nicht berechnet werden, da beide Beurteilenden alle CT mit dem Wert „5” beurteilten.

Compared to group A (control), group B1 showed significantly reduced supra- and infratentorial SNRs and supratentorial CNRs. The infratentorial CNRs were either significantly or almost significantly reduced in group B1.

When the ratio of ASIR blending was further increased to $40 \%$ in group B2, the CNRs were comparable to group A (except for infratentorial white matter/vermis CNR). SNR measures were similar. When ASIR blending was increased to $40 \%$ (group B2), the SNRs showed higher levels than in group B1 (20\% ASIR blending). Ventricular and infratentorial gray matter SNRs reached the control group levels in group B2, while the supratentorial gray and white matter as well as infratentorial white matter SNRs increased (compared to group B1) but did not reach the control group levels.

All CNRs and SNRs were significantly reduced in group C1 compared to control group A. When blending was increased to $50 \%$ (group C2) almost all SNR and CNR values improved slightly but did not reach levels comparable to group B2.

\section{Qualitative Analysis of Image Quality}

- Table 4, $\odot$ Fig. 2, 3 present the results of the qualitative analysis of image quality and interobserver agreement.

Compared to group A, image quality in terms of noise and supratentorial and infratentorial contrast were significantly reduced in group B1 and also in group B2, albeit to a lesser extent.

Overall diagnosability was slightly compromised in group B1 or B2. Group C1 showed significantly poorer results in terms of noise levels, contrast, diagnostic confidence and overall diagnosability com- pared to groups A and B1/2. Group C2 showed only slightly better results than group $\mathrm{C} 1$ without statistical significance.

Subgroup analysis of diagnostic confidence revealed no significant differences for patients suffering from acute bleeding between group B1/2 and group A. Comparing group C and group A, we found significantly lower values in group $C$ with difficulties in identifying subtle bleeding, such as small subarachnoid hemorrhage, whereas marked bleeding could sufficiently be detected. In patients with subacute stroke, values were significantly lower in group B1 compared to group A, but were comparable in group B2 (with ASIR blending increased to $40 \%$ ). Group C1 showed significantly lower values than the control group, and group C2 showed only marginally better results than group $\mathrm{C} 1$.

Image reconstruction-related artifacts were not seen in any of the evaluated groups.

\section{Radiation Dose}

Data on radiation doses are summarized in $\bullet$ Table 5, $\bullet$ Fig. 4. Using 20\% ASIR for the CT scan (group B1 and group B2) led to a significant reduction of the effective dose (ED) of $40.4 \%$ compared to group A. Using $30 \%$ ASIR during the scan (group C) reduced the ED by $73.3 \%$.

\section{Discussion}

With the number of emergency CT scans performed worldwide increasing constantly, there is a growing discussion on radia- 

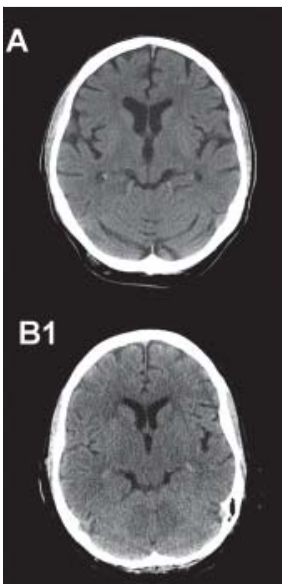

B2

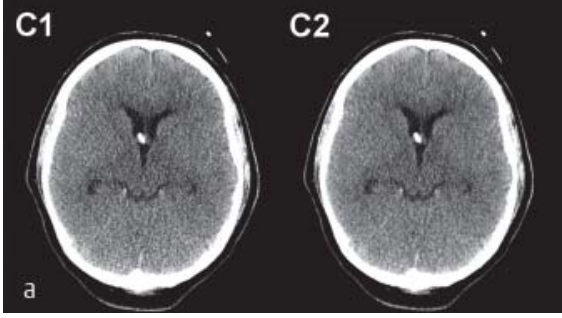

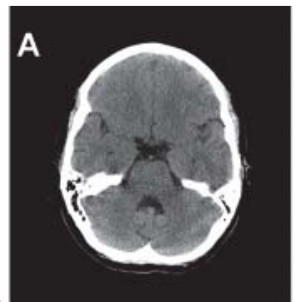

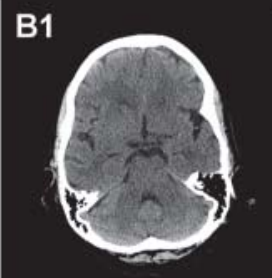

B2

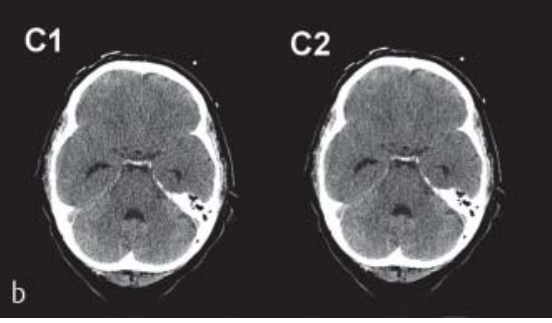

Fig. 2 Image quality of cCTs obtained in patients with no pathology. a Patients without acute or subacute pathology, supratentorial image quality. Scanning performed using $120 \mathrm{kV}$ and FBP (Group A), $20 \%$ ASIR (group B1), $20 \%$ ASIR for dose reduction and $40 \%$ ASIR/60\% FBP blending (group B2) and $30 \%$ ASIR (group C1) as well as $30 \%$ ASIR for dose reduction and $50 \%$ ASIR/50\% FBP blending (group C2). Note partially displayed ventricular drainage in group $\mathrm{C} 1 / 2$. b Patients without acute or subacute pathology, infratentorial image quality, groups A, B1, B2, C1 and C2.

Abb. 2 Bildqualität von cCT in Patienten ohne intracranielle Pathologie. a Patienten ohne akute oder subakute Pathologie, supratentorielle Bildqualität. CT durchgeführt mit $120 \mathrm{kV}$ und FBP (Gruppe A), $20 \%$ ASIR (Gruppe B1), $20 \%$ ASIR zur Dosisreduktion und $40 \%$ ASIR/60\% FBP Rekonstruktion (Gruppe B2) und 30\% ASIR (Gruppe C1) sowie 30\% ASIR zur Dosisreduktion und $50 \%$ ASIR/50 \% FBP Rekonstruktion (Gruppe C2). Angeschnittene Ventrikeldrainage in Gruppe C1/2. b Patienten ohne akute oder subakute Pathologie, infratentorielle Bildqualität, Gruppen A, B1, B2, C1 und C2.

Table 5 Total DLPs, CTDIvol and effective doses.

Tab. 5 Gesamt DLPs, CTDIvol und effektive Dosis.

\begin{tabular}{|c|c|c|c|c|c|c|c|c|c|}
\hline & group A & group B1 & A vs. $B 1$ & group B2 & A vs. $B 2$ & group C1 & A vs. $C 1$ & group C2 & A vs. $C 2$ \\
\hline & $120 \mathrm{kV} / \mathrm{FBP}$ & $120 \mathrm{kV} / A S I R 20$ & p-value & $\begin{array}{l}120 \mathrm{kV} / A S I R 20 \\
(40 \% / 60 \%)\end{array}$ & p-value & 120 kV/ASIR30 & p-value & $\begin{array}{l}120 \mathrm{kV} / A S I R 30 \\
(50 \% / 50 \%)\end{array}$ & p-value \\
\hline CTDIvol & $51.6 \pm 2.7$ & $30.2 \pm 2.9$ & $p<0.0001$ & $30.1 \pm 3$ & $p<0.0001$ & $13.9 \pm 6.28$ & $p<0.0001$ & $13.9 \pm 6.28$ & $\mathrm{p}<0.0001$ \\
\hline $\begin{array}{l}\text { total DLP } \\
(\mathrm{mGy} \times \mathrm{cm})\end{array}$ & $768 \pm 52$ & $455 \pm 55$ & $p<0.0001$ & $455 \pm 57$ & $p<0.0001$ & $204 \pm 97$ & $p<0.0001$ & $204 \pm 97$ & $\mathrm{p}<0.0001$ \\
\hline $\begin{array}{l}\text { effective dose } \\
\text { (mSv) }\end{array}$ & $1.61 \pm 0.11$ & $1.05 \pm 0.13$ & $p<0.0001$ & $0.96 \pm 0.11$ & $p<0.0001$ & $0.43 \pm 0.20$ & $p<0.0001$ & $0.43 \pm 0.20$ & $p<0.0001$ \\
\hline
\end{tabular}

Using $20 \%$ of ASIR (group B1 and group B2) led to a significant reduction in the ED of $40.4 \%$ compared to group A. Using $30 \%$ of ASIR during the scan (group C1 and group 2 ) reduced the ED by $73.3 \%$.

Die Anwendung von $20 \%$ ASIR (Gruppe B1 und Gruppe B2) führte zu einer signifikanten Reduktion der effektiven Dosis um 40,4\% im Vergleich zur Kontrollgruppe. Die Anwendung von $30 \%$ ASIR (Gruppe C1 und Gruppe C2) führte zu einer signifikanten Reduktion der effektiven Dosis um 73,3\%.

tion-associated risks [15]. In this study, approximately three quarters of patients referred for cranial CT from the first-aid department had no acute or subacute pathology and almost half of them did not show any pathology whatsoever. Due to the carcinogenic potential of ionizing radiation, cCTs should thus be performed with the lowest radiation dose that still allows adequate diagnosis especially when younger patients are examined.

The implementation of IR algorithms is particularly noteworthy in this context. Several studies have shown that IR algorithms significantly reduce dose while maintaining, or in some cases even improving, image quality [8, 9, 16 -18].

The results of our study show that use of a CT protocol with $20 \%$ ASIR reduces the dose of cranial CT by $40.4 \%$. When combined with blending of $40 \%$ ASIR/60\% FBP, supratentorial CNRs are comparable to those of the control group and infratentorial CNRs remain acceptable. Subjective quality levels, e.g. contrast, overall diagnosability and diagnostic confidence, are also still acceptable. We now routinely use this $\mathrm{CT}$ protocol in patients referred from the emergency department in our clinic.
A CT protocol with 30\% ASIR and an increased noise index degrades both quantitative and qualitative image quality to such an extent that it is unacceptable in everyday clinical practice. However, the quality remains high enough for the diagnosis of life-threatening conditions, such as acute bleeding, or brain edema or for the assessment of hydrocephalus especially when blending is increased to $50 \%$ ASIR/50\% FBP. In these cases, this protocol achieved sub-millisievert scanning $(0.43 \pm 0.20 \mathrm{mSv})$, which is particularly useful for the repeated follow-up examination of neurosurgical ICU patients.

One of the first studies investigating the use of ASIR in adult cranial CT was conducted by Kilic et al. [11]. In this study, the authors showed a $31 \%$ DLP reduction of cranial CT scans when $30 \%$ ASIR was applied during acquisition. There was no significant reduction in image quality and interpretability (adult patients, 49 FBP cCTs, 98 ASIR cCTs).

Ren et al. investigated the potential role of ASIR in cCTs of adults over 50 years of age. They showed a $30 \%$ dose reduction in 200 mAs cCTs with 50\% ASIR blending compared to 300 mAs cCTs 

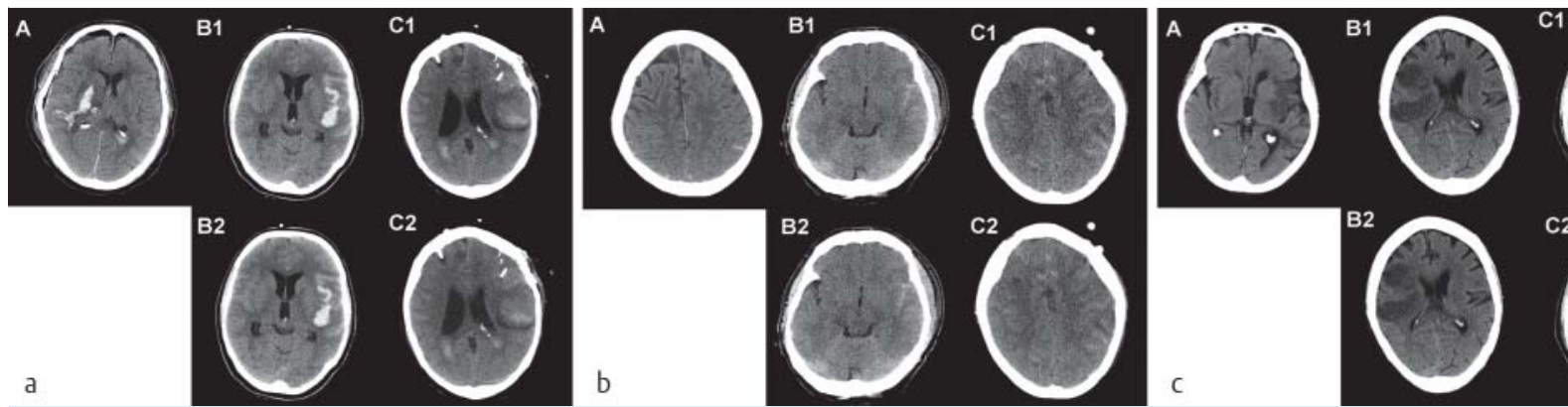

C2

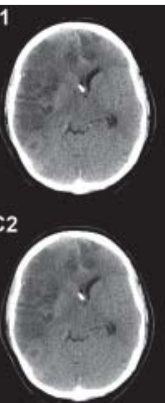

Fig.3 Image quality of $\mathrm{CCTs}$ obtained in patients with acute pathologies. a Patients suffering from massive intracranial bleeding (ICB). ICB could easily be diagnosed in all groups. b Patients suffering from subarachnoid hemorrhage $(S A B)$. More subtle bleeding like $S A B$ could easily be diagnosed in group B1/2 and in the control group. Diagnostic confidence was lower in group C $1 / 2$ compared to the control group but still acceptable for follow-up imaging. c Patients suffering from subacute stroke. Subjective quality was significantly lower in group B1 than in group A. The subjective quality in group B2 (when ASIR blending was increased to $40 \%$ ) was comparable to group A. Group C1/2 showed significantly lower values than the control group but was considered acceptable for follow-up imaging.
Abb. 3 Bildqualität der cCT von Patienten mit akuter intracranieller Pathologie. a Patienten mit intracranieller Blutung (ICB). Die Diagnose der massiven ICB kann in allen Gruppen problemlos gestellt werden. b Patienten mit subarachnoider Blutung (SAB). Subtilere Blutungen wie SAB konnten problemlos in den Gruppen $A$ und B1/2 diagnostiziert werden; der diagnostische Wert der Bilder in den Gruppen C1/2 war niedriger aber ausreichend für Folge-CT. c Patienten mit subakutem Schlaganfall. Die subjektive Bildqualität der Gruppe B1 war niedriger als in der Kontrollgruppe. Die subjektive Qualität in Gruppe B2 (40\% ASIR Rekonstruktion) war vergleichbar mit Gruppe A. Gruppe C1/2 zeigte signifikant niedrigere Werte als die Kontrollgruppe, war aber ausreichend für Folge-CT.

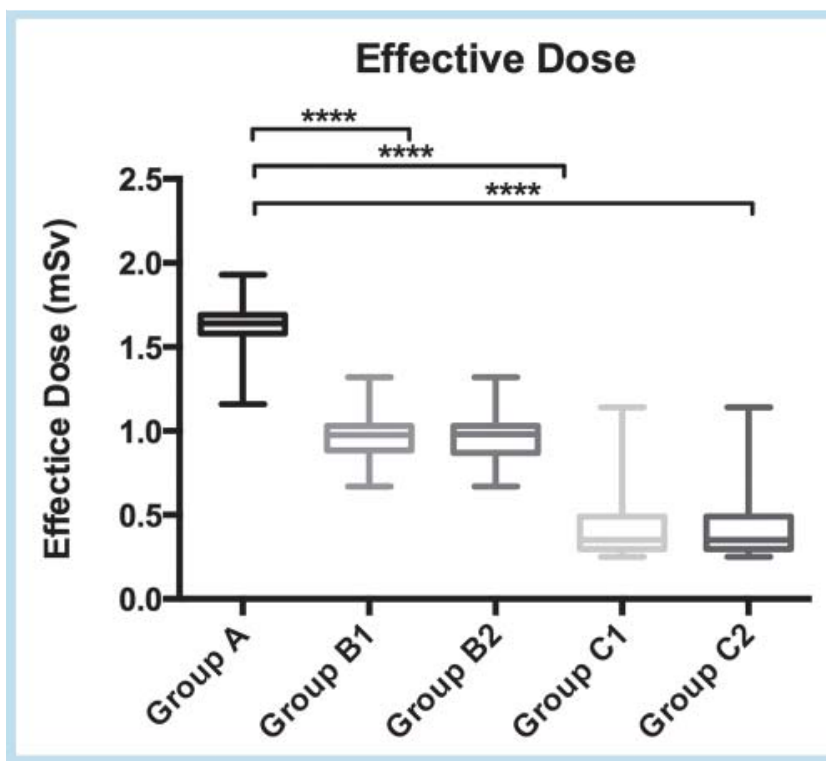

Fig. 4 Effective dose (ED) in cranial CTs using $120 \mathrm{kV}$ and $F B P(A), 120 \mathrm{kV}$ and $20 \%$ ASIR (B1 / B2) and $30 \%$ ASIR (C1/2). Using $20 \%$ of ASIR (group B1 and group B2) led to a significant reduction of ED of $40.4 \%$ compared to group A. Using $30 \%$ of ASIR during the scan (group C $1 / 2$ ) reduced the ED by $73.3 \%$.

Abb. 4 Die effektive Dosis (ED) cranialer CTs mit $120 \mathrm{kV}$ und FBP (A), $120 \mathrm{kV}$ und $20 \%$ ASIR (B1 / B2) und $30 \%$ ASIR (C1/2). $20 \%$ ASIR (Gruppe B1 und Gruppe B2) führte zu einer signifikanten Reduktion der ED um 40,4\% im Vergleich zur Gruppe A. 30 \% ASIR (Gruppe C1/2) führte zu einer Reduktion der ED um $73,3 \%$.

with FBP reconstruction (age $>50 y, 40$ patients) [19]. A reduction of the tube current time product from $300 \mathrm{mAs}$ to $200 \mathrm{mAs}$ roughly corresponds to the use of $30 \%$ ASIR default settings during the scan. In our study, the use of $30 \%$ ASIR led to a higher dose reduction of $73.3 \%$. A possible explanation could be the use of different noise indices. Unfortunately, NIs were not reported by Ren et al. They evaluated diagnostic confidence but provided no information on detected pathologies. The authors state that they focused on chronic vascular cerebral disease when scoring image quality. Korn and colleagues examined objective and subjective image quality at reduced tube current rates in sinogram-affirmed iterative reconstruction (SAFIRE) cCTs compared to standard dose FBP cCTs (320 mAs vs. $255 \mathrm{mAs}$ ). At a $20 \%$ dose reduction, reconstruction of a head CT by SAFIRE provided better objective and subjective image quality than FBP reconstruction (30 FBP cCTs, 30 SAFIRE cCTs) [12]. The main purpose of this study was not to reduce the dose while maintaining image quality but to improve image quality while maintaining the dose.

Haubenreisser et al. assessed objective and subjective image quality in FBP and SAFIRE-reconstructed cCTs of different slice widths ( $1-5 \mathrm{~mm}$; $1 \mathrm{~mm}$ increments). They showed significant reductions in image noise and improved subjective image particularly in thinner slices (29 patients, 40 cCTs) [20]. This small study, similar to the work of Korn et al., focused on finding the best reconstruction parameters at a certain dose level and did not aim at dose reduction.

To our knowledge, the largest and most sophisticated study on iterative reconstruction to date was performed by Komlosi et al., who investigated $200 \mathrm{cCTs}$ and showed that use of an NI of 5 (compared to FBP and an NI of 4) and 40\% ASIR blending led to a $10.5 \%$ reduction in DLPs in adult cCTs while the image quality and noise were comparable (100 FBP cCTs, 100 ASIR cCTs) [21]. Similar to our study, the authors gradually increased the NI and then used different levels of ASIR/FBP blending to compensate for the higher NI. While the extent of work is impressive, it is unfortunate that the authors did not analyze SNRs or CNRs in the brain, which makes it hard to objectively judge image quality and noise in certain brain regions. This is especially problematic since we believe that image quality in infratentorial regions might be more dependent on dose variations during the scan due to the higher bone thickness in the region. Also, an analysis of the frequency of different pathologies was not performed.

\section{Strengths and Limitations}

To our knowledge, the work presented here is one of the largest studies investigating iterative reconstruction in cranial CT. De- 
spite the relatively high number of scans performed, no study in this field has yet put an emphasis on emergency department patients or analyzed the frequency of different pathologies. Also, no earlier investigators have performed subgroup analysis of different pathologies. Finally, it has to be mentioned that other publications have not distinguished between infra- and supratentorial image quality in subjective and objective image analysis.

Our study has several limitations. Firstly, no explicit patient group matching was done. However, the patient parameters matched well in terms of age, gender or head diameters.

Secondly, image quality evaluation was based on the subjective impression of two readers and qualitative analysis may indeed not have been completely blind, since an experienced radiologist may identify an ASIR image by its typical appearance. However, we also performed objective quantitative image analysis to corroborate qualitative evaluation. Nevertheless, it has been questioned whether quantitative measures are the appropriate tool for evaluating the effectiveness of IR algorithms. Jensen et al. showed that lesion detection in a liver phantom was not improved in ASIR-reconstructed images compared to FBP-reconstructed images of a liver phantom even though the noise decreased and the CNR increased significantly [22].

Thirdly, patients with foreign material in the skull were excluded in groups $A$ and $B 1 / 2$ but not in group $C 1 / 2$, which might have influenced the noise levels in group $\mathrm{C} 1 / 2$.

\section{Conclusion}

IR algorithms are a promising option for reducing radiation exposure without compromising image quality in cranial CT. A CT protocol with a combination of $20 \%$ ASIR and a $40 \%$ ASIR/ $60 \%$ FBP blending ratio decreases the effective dose significantly by $40.4 \%$, while producing scans with similar image quality compared to a routine dose $\mathrm{cCT}$. This $\mathrm{CT}$ protocol is recommended for everyday clinical practice in an emergency department setting. The use of a CT protocol with $30 \%$ ASIR and $50 \%$ ASIR/50\% FBP reduces the effective dose by $73.3 \%$ and can be considered for followup scans of neurosurgical ICU patients.

\section{Clinical Relevance of the Study}

- The use of computed tomography has been constantly increasing and leads to higher doses of ionizing radiation in the population

- The routine use of $20 \%$ ASIR cCTs with $40 \%$ ASIR/ $60 \%$ FBP blending may lead to a dose reduction of more than $40 \%$ in these cCTs without compromising diagnosis-related confidence

- $30 \%$ ASIR cCTs with $50 \%$ ASIR/50\%FBP are adequate for follow-up imaging and offer a dose reduction of over $70 \%$ in these cCTs

\section{References}

1 Hall EJ, Brenner DJ. Cancer risks from diagnostic radiology. The British journal of radiology 2008; $81: 362-378$

2 [Anonymous]. Radiation exposures from medical applications. In. Salzgitter: Bundesamt für Strahlenschutz; 2012
3 Pearce MS, Salotti JA, Little MP et al. Radiation exposure from CT scans in childhood and subsequent risk of leukaemia and brain tumours: a retrospective cohort study. Lancet 2012; 380: 499-505

4 Kalender WA, Wolf $H$, Suess $C$. Dose reduction in CT by anatomically adapted tube current modulation. II. Phantom measurements. Medical physics 1999; 26: $2248-2253$

5 McCollough CH, Bruesewitz MR, Kofler JM et al. CT dose reduction and dose management tools: overview of available options. Radiographics: a review publication of the Radiological Society of North America, Inc 2006; 26: $503-512$

6 Kilic K, Erbas G, Guryildirim $M$ et al. Quantitative and qualitative comparison of standard-dose and low-dose pediatric head computed tomography: a retrospective study assessing the effect of adaptive statistical iterative reconstruction. Journal of computer assisted tomography 2013; 37: $377-381$

7 Alkim E, Gurbuz E, Kilic E. A fast and adaptive automated disease diagnosis method with an innovative neural network model. Neural networks: the official journal of the International Neural Network Society 2012; 33: 88 - 96

8 Willemink MJ, de Jong PA, Leiner T et al. Iterative reconstruction techniques for computed tomography Part 1: technical principles. European radiology 2013; 23: $1623-1631$

9 Willemink MJ, Leiner T, de Jong PA et al. Iterative reconstruction techniques for computed tomography part 2 : initial results in dose reduction and image quality. European radiology 2013; 23: 1632 - 1642

10 Wu TH, Hung SC, Sun JY et al. How far can the radiation dose be lowered in head CT with iterative reconstruction? Analysis of imaging quality and diagnostic accuracy. European radiology 2013; 23: 2612-2621

11 Kilic K, Erbas G, Guryildirim M et al. Lowering the dose in head CT using adaptive statistical iterative reconstruction. AJNR American journal of neuroradiology 2011; 32: 1578 - 1582

12 Korn A, Bender B, Fenchel $M$ et al. Sinogram affirmed iterative reconstruction in head CT: improvement of objective and subjective image quality with concomitant radiation dose reduction. European journal of radiology 2013; 82: 1431 - 1435

13 Mueck FG, Korner M, Scherr MK et al. Upgrade to iterative image reconstruction (IR) in abdominal MDCT imaging: a clinical study for detailed parameter optimization beyond vendor recommendations using the adaptive statistical iterative reconstruction environment (ASIR). RoFo: Fortschritte auf dem Gebiete der Rontgenstrahlen und der Nuklearmedizin 2012; 184: 229-238

14 [Anonymous]. The measurement, reporting, and management of radiation dose in CT: report of AAPM Task Group 23 of the Diagnostic Imaging Council CT Committee. In, AAPM report no 96: American Association of Physicists in Medicine; 2008

15 Smith-Bindman $R$, Lipson J, Marcus $R$ et al. Radiation dose associated with common computed tomography examinations and the associated lifetime attributable risk of cancer. Archives of internal medicine 2009; 169: $2078-2086$

16 Kaul D, Grupp U, Kahn J et al. Reducing radiation dose in the diagnosis of pulmonary embolism using adaptive statistical iterative reconstruction and lower tube potential in computed tomography. European radiology 2014; 24: 2685 - 2691

17 Kahn J, Grupp U, Rotzinger R et al. CT for evaluation of potential renal donors - how does iterative reconstruction influence image quality and dose? European journal of radiology 2014; 83: $1332-1336$

18 Grupp U, Schafer ML, Meyer H et al. Reducing Radiation Dose in Emergency CT Scans While Maintaining Equal Image Quality: Just a Promise or Reality for Severely Injured Patients? Emergency medicine international 2013; 2013: 984645

19 Ren Q Dewan SK, Li M et al. Comparison of adaptive statistical iterative and filtered back projection reconstruction techniques in brain CT. European journal of radiology 2012; 81: 2597-2601

20 Haubenreisser H, Fink C, Nance JW Jr et al. Feasibility of slice width reduction for spiral cranial computed tomography using iterative image reconstruction. European journal of radiology 2014; 83: 964-969

21 Komlosi $P$, Zhang $Y$, Leiva-Salinas $C$ et al. Adaptive statistical iterative reconstruction reduces patient radiation dose in neuroradiology CT studies. Neuroradiology 2014; 56: 187 -193

22 Jensen K, Martinsen AC, Tingberg A et al. Comparing five different iterative reconstruction algorithms for computed tomography in an ROC study. European radiology 2014; 24: 2989-3002 